\section{Review: benzodiazepines increase sleep duration but also lead to adverse effects in adults with insomnia}

Holbrook AM, Crowther R, Lotter A, et al. Meta-analysis of benzodiazepine use in the treatment of insomnia. CMAJ 2000 Jan 25;162:225-33.

QUESTION: In adults with insomnia, are benzodiazepines effective and safe for improving sleep outcomes?

\section{Data sources}

Studies were identified by searching Medline (1966-98), the Cochrane Controlled Trials Register, and reference lists, and contacting manufacturers of benzodiazepines.

\section{Study selection}

English language studies were selected if they were randomised controlled trials (RCTs) that compared a benzodiazepine with a placebo or another active drug in patients with insomnia.

\section{Data extraction}

RCTs were assessed for the quality of study methods. Data were extracted on study design, conditions treated, patient characteristics, setting and duration of RCT, and outcomes (including adverse effects).

\section{Main results}

45 RCTs (2672 patients) (mean age range 29-82 y, 53\% men) met the selection criteria. Study duration ranged from 1 day to 6 weeks (median 7.5 d). Patients who received benzodiazepines had greater sleep duration than those who received placebo (2 RCTs) (table). A statistically non-significant decrease occurred in benzodiazepine group sleep latency (8 comparisons in 4 RCTs) (table). Patient estimates of sleep latency were shorter in the benzodiazepine group than in the placebo group (8 RCTs), but heterogeneity existed. When RCTs were grouped by quality of study methods, heterogeneity was no longer statistically significant; the effect was smaller for high quality RCTs than for low quality RCTs (table). Patient estimates of sleep duration were greater in the benzodiazepine group than in the placebo group (8 RCTs) (table). Overall adverse effects (7 RCTs) and daytime drowsiness (8 RCTs) were more common in the benzodiazepine group than in the placebo group; dizziness or lightheadedness was also more common in the benzodiazepine group (4 RCTs), but this difference was not statistically significant (table). Zopiclone was not superior to benzodiazepines on any outcome measured.

\section{Conclusion}

Benzodiazepines improve sleep duration but also lead to adverse effects in adults with insomnia.

\section{COMMENTARY} of these problems. 1997;20:1111-8 in persistent sleep-onset insomnia. Am J Psychiatry 1991;148:121-6. life insomnia: a randomized controlled trial. JAMA 1999;281:991-9. demiol 1995;5:239-44. time factor. Age Ageing 1996;25:273-8.
Sources of funding: in

part, Canadian

Pharmaceutical

Association and

Canadian Medical

Association.

For correspondence: Dr A M Holbrook, Centre for Evaluation of Medicines, St Joseph's Hospital, 50 Charlton Avenue East, Hamilton, Ontario L8N 4A6, Canada.Fax +1905 5216136.

What information do we need to consider when treating patients with insomnia? We need to know (1) by how much a medication is better than placebo or non-pharmacological treatment for improving objective measures of sleep, subjective quality of sleep, and overall quality of life; and (2) by how much that medication is worse than placebo or non-pharmacological treatment with respect to drowsiness, cognitive impairment, dependence, tolerance, accident proneness, teratogenicity, and cost. Furthermore, we need to know these estimates not only for several days but also for much longer periods of time.

After reading this methodologically rigorous, comprehensive meta-analysis by Holbrook et al, are we in a better position to answer these questions? Firstly, this meta-analysis does not show how benzodiazepines affect the patients' quality of sleep or quality of life because these were not measured in the original RCTs. Secondly, no comparison between benzodiazepines and non-pharmacological alternatives was presented. Finally, none of the meta-analysed RCTs examined long term use. The authors note all

Importantly, a few RCTs are now available that directly compare pharmacotherapy and non-pharmacological treatment. These studies suggest a greater commitment of time, but equal effectiveness and superior durability for behavioural techniques. ${ }^{1-3}$ Some problems of benzodiazapine pharmacotherapy can now be quantified. A cohort study suggested that approximately 1 in 2900 people will be admitted to hospital for a traffic accident within 2 weeks after their first prescription of a benzodiazepine. ${ }^{4}$ For every 100 elderly people who take benzodiazepines, approximately 1 person is admitted to hospital for a fall.

As a clinician, I must confess that it is difficult to balance this information against an approximate 1 hour increase in total sleep time as well as increased drowsiness and dizziness as shown by this meta-analysis. I concur with the authors that we need further pragmatic studies before we can make strong evidence-based recommendations. Toshiaki Furukawa, MD, PhD Nagoya City University Nagoya, Japan

1 Hauri PJ. Can we mix behavioral therapy with hypnotics when treating insomniacs? Sleep

2 McClusky HY, Milby JB, Switzer PK, et al. Efficacy of behavioral versus triazolam treatment

3 Morin CM, Colecchi C, Stone J, et al. Behavioral and pharmacological therapies for late-

4 Neutel CI. Risk of traffic accident injury after a prescription for a benzodiazepine. Ann Epi-

5 Neutel CI, Hirdes JP, Maxwell CJ, et al. New evidence on benzodiazepine use and falls: the

Benzodiazepines $v$ placebo in adults with insomnia*

\begin{tabular}{|c|c|c|c|c|}
\hline Outcomes & No of comparisonst & & No of patients & $\begin{array}{l}\text { Weighted mean difference } \\
(95 \% \mathrm{CI})\end{array}$ \\
\hline Sleep latency (min) & 8 & & 159 & $-4.2(-0.7$ to 9.2$) \ddagger$ \\
\hline Total sleep duration (min) & 3 & & 35 & $61.8(37.4$ to 86.2$)$ \\
\hline Patient estimated sleep latency, high quality studies (min) & $7 \S$ & & $379 \S$ & $-11.7(-7.6$ to -15.8$)$ \\
\hline Patient estimated sleep latency, low quality studies (min) & $6 \S$ & & $136 \S$ & $-23.7(-15.8$ to -31.5$)$ \\
\hline Patient estimated sleep duration (min) & $13 \S$ & & 566 & $48.4(39.6$ to 57.1$)$ \\
\hline Outcomes & No of comparisonst & No of patients & Weighted RRI $(\mathbf{C l})^{\star *}$ & $\mathrm{NNH}^{* *}$ \\
\hline Adverse effects & 11 & 821 & $32 \%$ (17 to 49 ) & 8 \\
\hline Daytime drowsiness & $13 \S$ & 889 & $76 \%$ (43 to 117$)$ & 8 \\
\hline Dizziness or lightheadedness & $5 \S$ & 326 & $97 \%$ (-24 to 413$)$ & Not significant \\
\hline
\end{tabular}

*Study duration ranged from 1 day to 6 weeks. †Some studies compared $>1$ type of benzodiazepine with placebo.

‡Not significant. §Data provided by author. $\quad{ }^{* *}$ Abbreviations defined in glossary; RRI, $\mathrm{NNH}$, and $\mathrm{Cl}$ provided by author. 